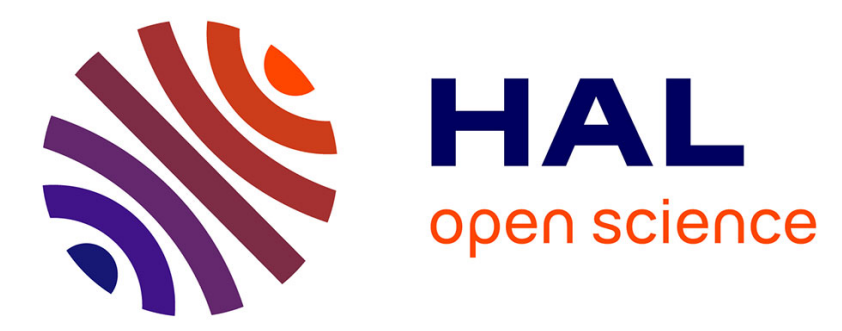

\title{
Structural, thermal, and electrical properties of $\mathrm{CrSi} 2$
} T. Dasgupta, Jean Etourneau, Bernard Chevalier, Samir F. Matar, A. M. Umarji

\section{To cite this version:}

T. Dasgupta, Jean Etourneau, Bernard Chevalier, Samir F. Matar, A. M. Umarji. Structural, thermal, and electrical properties of CrSi2. Journal of Applied Physics, 2008, 103 (11), pp.113516. 10.1063/1.2917347 . hal-00292837

\section{HAL Id: hal-00292837 https://hal.science/hal-00292837}

Submitted on 3 Nov 2021

HAL is a multi-disciplinary open access archive for the deposit and dissemination of scientific research documents, whether they are published or not. The documents may come from teaching and research institutions in France or abroad, or from public or private research centers.
L'archive ouverte pluridisciplinaire HAL, est destinée au dépôt et à la diffusion de documents scientifiques de niveau recherche, publiés ou non, émanant des établissements d'enseignement et de recherche français ou étrangers, des laboratoires publics ou privés. 


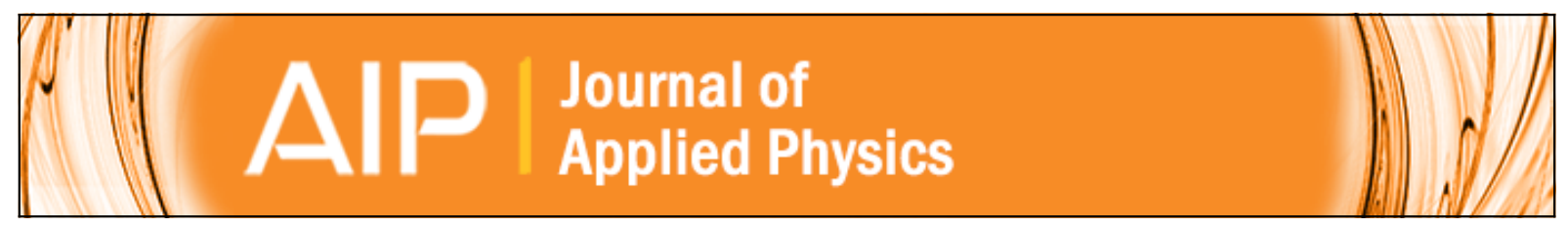

\section{Structural, thermal, and electrical properties of $\mathrm{Cr} \mathrm{Si} 2$}

T. Dasgupta, J. Etourneau, B. Chevalier, S. F. Matar, and A. M. Umarji

Citation: Journal of Applied Physics 103, 113516 (2008); doi: 10.1063/1.2917347

View online: http://dx.doi.org/10.1063/1.2917347

View Table of Contents: http://scitation.aip.org/content/aip/journal/jap/103/11?ver=pdfcov

Published by the AIP Publishing

\section{Articles you may be interested in}

Thermoelectric properties of the Heusler-type F e $2 \mathrm{~V} \mathrm{~T}$ a x A I $1-x$ alloys

J. Appl. Phys. 115, 033707 (2014); 10.1063/1.4861419

Cross-plane electronic and thermal transport properties of p-type La0.67Sr0.33MnO3/LaMnO3 perovskite oxide metal/semiconductor superlattices

J. Appl. Phys. 112, 063714 (2012); 10.1063/1.4754514

Transport properties of the clathrate BaGe5

J. Appl. Phys. 110, 043715 (2011); 10.1063/1.3625233

Correlation between structural and low-temperature thermoelectric properties of $\mathrm{Zn} 13+\mathrm{x}$ Sb 10 compounds J. Appl. Phys. 105, 013713 (2009); 10.1063/1.3043884

Magnetic and thermoelectric properties of R $3 \mathrm{Cu} 3 \mathrm{Sb} 4$ ( R=La , Ce, Gd, Er) J. Appl. Phys. 83, 2568 (1998); 10.1063/1.367018

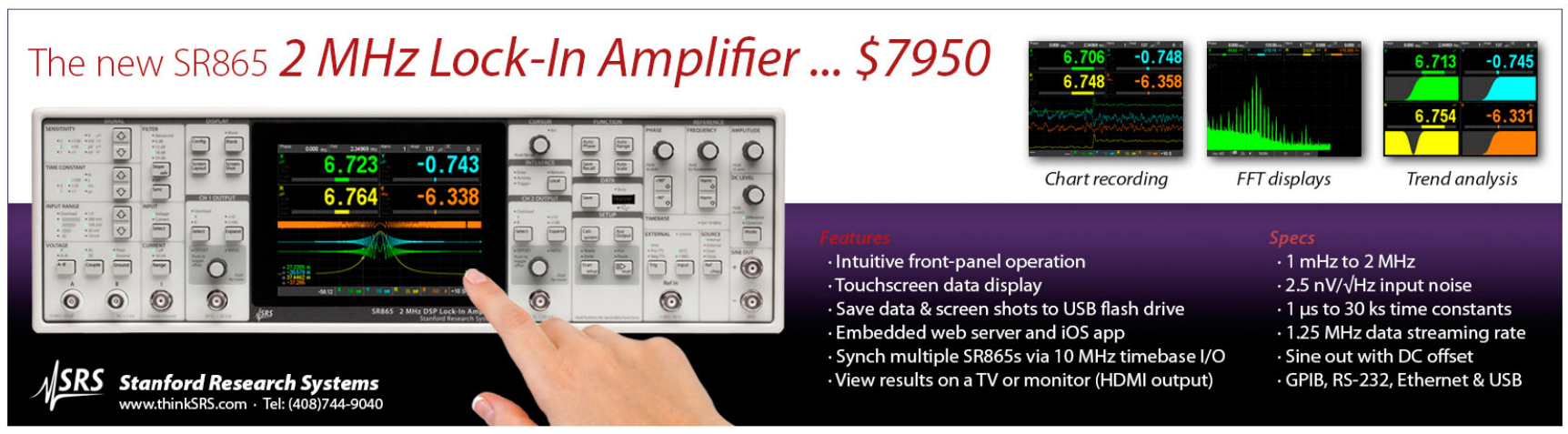




\title{
Structural, thermal, and electrical properties of $\mathrm{CrSi}_{2}$
}

\author{
T. Dasgupta, ${ }^{1}$ J. Etourneau, ${ }^{2}$ B. Chevalier, ${ }^{2}$ S. F. Matar, ${ }^{2}$ and A. M. Umarji ${ }^{1, a)}$ \\ ${ }^{1}$ Materials Research Centre, Indian Institute of Science, Bangalore-560012, India \\ ${ }^{2}$ ICMCB, CNRS, Université Bordeaux 1, 87 Av. A. Schweitzer, 33608 Pessac Cedex, France
}

(Received 19 October 2007; accepted 6 March 2008; published online 12 June 2008)

\begin{abstract}
Stoichiometric $\mathrm{CrSi}_{2}$ was prepared by arc melting and compacted by uniaxial hot pressing for property measurements. The crystal structure of $\mathrm{CrSi}_{2}$ was investigated using the powder x-ray diffraction method. From the Rietveld refinement, the lattice parameters were found to be $a$ $=4.42757$ (7) and $c=6.36804$ (11) $\AA$, respectively. The thermal expansion measurement revealed an anisotropic expansion in the temperature range from room temperature $800 \mathrm{~K}$ with $\alpha_{a}=14.58$ $\times 10^{-6} / \mathrm{K}, \quad \alpha_{c}=7.51 \times 10^{-6} / \mathrm{K}$, and $\alpha_{V}=12.05 \times 10^{-6} / \mathrm{K}$. The volumetric thermal expansion coefficient shows an anomalous decrease in the temperature range of 450-600 K. The measured electrical resistivity $\rho$ and thermoelectric power $S$ have similar trends with a maxima around $550 \mathrm{~K}$. Thermal conductivity measurements show a monotonic decrease with increasing temperature from a room temperature value of $10 \mathrm{~W} \mathrm{~m}^{-1} \mathrm{~K}^{-1}$. The $\mathrm{ZT}$ values increase with temperature and have a maximum value of 0.18 in the temperature range studied. An analysis of the electronic band structure is provided. (C) 2008 American Institute of Physics. [DOI: 10.1063/1.2917347]
\end{abstract}

\section{INTRODUCTION}

Transition metal (TM) silicides are potential materials for different high temperature applications due to their high melting points and chemical stability at elevated temperatures. ${ }^{1,2}$ For thermoelectric power generation which requires high efficiency at elevated temperatures, $\mathrm{CrSi}_{2}$ is a possible candidate. ${ }^{3,4}$ This is because of its semiconducting nature ${ }^{5}$ and thermal stability in air up to $1000 \mathrm{~K}^{6} \mathrm{CrSi}_{2}$ has a $C 40$ hexagonal structure with space group $\mathrm{Pb}_{2} 22$. The crystal lattice consists of $\mathrm{Cr}$ atoms in $3 d$ positions and $\mathrm{Si}$ atoms at $6 j$ positions. The position of $\mathrm{Si}$ atoms is expressed with the parameter $x .^{7}$ Both first principle calculations and experimental single crystal diffraction data reveal a deviation of Si positions $(x)$ from the ideal value $(1 / 6)$ for different TM disilicides. ${ }^{8,9}$ Any deviation of the $\mathrm{Si}$ atoms breaks the sixfold coordination of the TM atoms and hence can affect the thermal and transport properties of the compound.

Stoichiometric $\mathrm{CrSi}_{2}$ is reported to have an indirect band gap of $0.35 \mathrm{eV}$ confirmed from both experimental works and theoretical calculations. ${ }^{10-12}$ Its room temperature (RT) value of the Seebeck coefficient $(S)$ is $\sim 100 \mu \mathrm{V} / \mathrm{K}$ and the electrical resistivity $(\rho=1 / \sigma)$ is of the order of $10^{-3} \Omega \mathrm{cm} .{ }^{5}$ The thermal conductivity $(K)$ value at RT is $\sim 10 \mathrm{~W} / \mathrm{mK}$ with the phonons being the major contributors. It is the high $K$ value in this material, which limits its thermoelectric figure of merit (ZT), defined as

$$
\mathrm{ZT}=\frac{S^{2} \sigma}{K} T
$$

where $T$ stands for temperature.

Attempts to improve $\mathrm{ZT}$ in $\mathrm{CrSi}_{2}$ both by doping and grain size reduction are reported in the literature. ${ }^{5,13,14} \mathrm{Re}-$ cently, substitution studies in both $\mathrm{Cr}$ and $\mathrm{Si}$ sites have

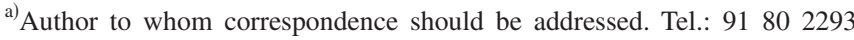
2944. FAX: 91802360 7316. Electronic mail: umarji@mrc.iisc.ernet.in.
}

shown a marginal improvement in thermoelectric performance. ${ }^{15,16}$ In order to achieve better thermoelectric efficiency in this material, it is thus necessary to relate the structure with properties in this material.

In this paper, the structural refinement and measurement of different thermal and electrical properties relevant to the thermoelectric performance have been carried out on stoichiometric $\mathrm{CrSi}_{2}$. Further, we complement the experimental results with electronic structure analyses of the band structure in the framework of the density functional theory.

\section{EXPERIMENTAL}

\section{A. Synthesis}

Synthesis was carried out by arc melting high purity $\mathrm{Cr}$ and $\mathrm{Si}$ pieces in the desired stoichiometric ratio. Titanium was first melted to getter oxygen, after which the sample was melted in flowing argon. Samples were remelted twice to achieve better homogenization. The resulting ingots were then crushed in an agate pestle and mortar and passed through a 325 mesh sieve to obtain fine powders. The powders were used for diffraction studies. For property measurements the powder was compacted by hot uniaxial pressing in BN coated graphite dye at $1450 \mathrm{~K}$ under $25 \mathrm{MPa}$ uniaxial pressure for a duration of $15 \mathrm{~min}$. The density of the compacted pellets was measured by the Archimedes method. Scanning electron micrographs (SEM) (FEI Quanta 200) were taken on fractured surfaces parallel to the pressing direction.

Thermal stability was checked from thermogravimetric analysis (TA Instruments-SDT Q600) using a powdered sample. Approximately $25 \mathrm{mg}$ of the sieved sample was used for the experiment. A heating rate of $10 \mathrm{~K} / \mathrm{min}$ was maintained and the experiment was performed in flowing air. 


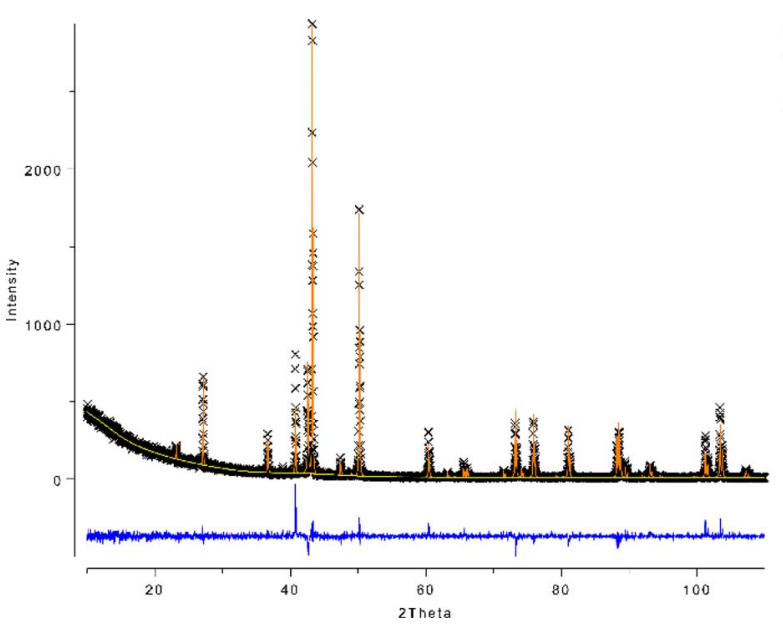

FIG. 1. (Color online) Rietveld refinement pattern for $\mathrm{CrSi}_{2}$. The observed and the calculated intensities and their differences are shown in the figure.

\section{B. X-ray diffraction}

Rietveld quality x-ray data were collected using $\mathrm{Cu} K \alpha$ radiation on a Philips (Xpert PRO) machine fitted with an accelerated detector (PW 3050/60). A step size of 0.017 and an effective time per step of $101.4 \mathrm{~s}$ were used during the measurement in the $2 \theta$ range of $10^{\circ}-110^{\circ}$. The average full widths at half maximum (FWHMs) of the peaks were 0.08 . The structural refinements were carried out using the software GSAS. ${ }^{17}$ High temperature x-ray data were collected using Co $K \alpha$ radiation in the temperature range from RT to $800 \mathrm{~K}$ at intervals of $40 \mathrm{~K}$. The calculation of the lattice parameters at each temperature was done using the lattice refinement program PROSZKI. ${ }^{18}$ From the data of the lattice parameters the thermal expansion $(\alpha)$ was calculated using the following formula:

$$
\alpha=\frac{1}{V} \frac{\Delta V}{\Delta T} \text {. }
$$

\section{Measurement of $S, K$, and $\sigma$}

The Seebeck coefficient was measured in a high temperature apparatus with respect to Platinum blocks. The details of the cell used and measurement methods have been given previously. ${ }^{19}$ The electrical resistivity was measured by a linear four-probe method in an inert atmosphere. The thermal diffusivity was measured by a commercial Netsch Laser flash apparatus in a $\mathrm{SiC}$ sample holder.

\section{RESULTS AND DISCUSSION}

\section{A. Structure refinement}

The crystal structure of $\mathrm{CrSi}_{2}$ was refined using the Rietveld method. The space group was assigned as $\mathrm{Pb}_{2} 22$ as reported in the literature. The results of the Rietveld refinement are shown in Fig. 1 (the crosses indicate the observed reflection profile and the solid line on top of it indicates the calculated one). The difference between the observed and the calculated lines is shown in the lower part of Fig. 1. The total number of measured Bragg reflections was 86 with the peak intensity of (111) being the largest. The results of the refined
TABLE I. Refined structural parameters of $\mathrm{CrSi}_{2}$

\begin{tabular}{|c|c|c|c|c|c|c|}
\hline \multicolumn{2}{|c|}{ Space Group } & \multicolumn{3}{|c|}{$a(\AA)$} & \multicolumn{2}{|c|}{$c(\AA)$} \\
\hline \multicolumn{2}{|c|}{$\mathrm{P}_{2} 22$} & \multicolumn{3}{|c|}{$4.42758(7)$} & \multicolumn{2}{|c|}{$6.36805(11)$} \\
\hline Atom & Site & $x$ & $y$ & $z$ & Occupancy & $U_{\text {iso }}$ \\
\hline $\mathrm{Cr}$ & $3 d$ & $1 / 2$ & 0 & $1 / 2$ & 1.001 & 0.00304 \\
\hline $\mathrm{Si}$ & $6 j$ & 0.165591 & 0.331183 & $1 / 2$ & 0.9870 & 0.00481 \\
\hline
\end{tabular}

lattice parameters, atomic positions, isotropic thermal parameters and occupancy are summarized in Table $I$. The $R_{P}$ value obtained was 0.08 and the $\mathrm{chi}^{2}$ value was 1.3 indicating a satisfactory fit. From Table I, it is observed that the $x$ coordinate of $\mathrm{Si}$ is 0.165 591, which is a slight deviation from the ideal geometry of $1 / 6$, expected in this compound. The $c / a$ ratio calculated from the refined lattice parameters is 1.438 , which is a deviation from the ideal value of 1.5 , resulting in interlayer bond distances that are smaller than the corresponding intralayer values. The theoretical calculations reported by Mattheiss ${ }^{12}$ indicated that the band gap in $\mathrm{CrSi}_{2}$ is very sensitive to the $c / a$ ratio and is seen to decrease with the reduction of this ratio. Thus transport properties that depend on the electronic band structure can vary depending on the $c / a$ ratio which, in turn, can be tuned by suitable substitutions in both $\mathrm{Cr}$ and $\mathrm{Si}$ sites.

\section{B. High temperature XRD}

The thermal dependence of the unit cell parameters and volume for $\mathrm{CrSi}_{2}$ is shown in Fig. 2. It is observed that the rate of increase of the $c$ parameter with temperature has a distinct reduction around $500 \mathrm{~K}$. This temperature regime also corresponds to the change in behavior of the transport properties as described in the latter section. The average thermal expansion in the temperature range studied $(300-775 \mathrm{~K})$ are $\alpha_{a}=14.58 \times 10^{-6} / \mathrm{K}, \alpha_{c}=7.51 \times 10^{-6} / \mathrm{K}$, and $\alpha_{V}=12.05 \times 10^{-6} / \mathrm{K}$. Thus there is a large anisotropy in

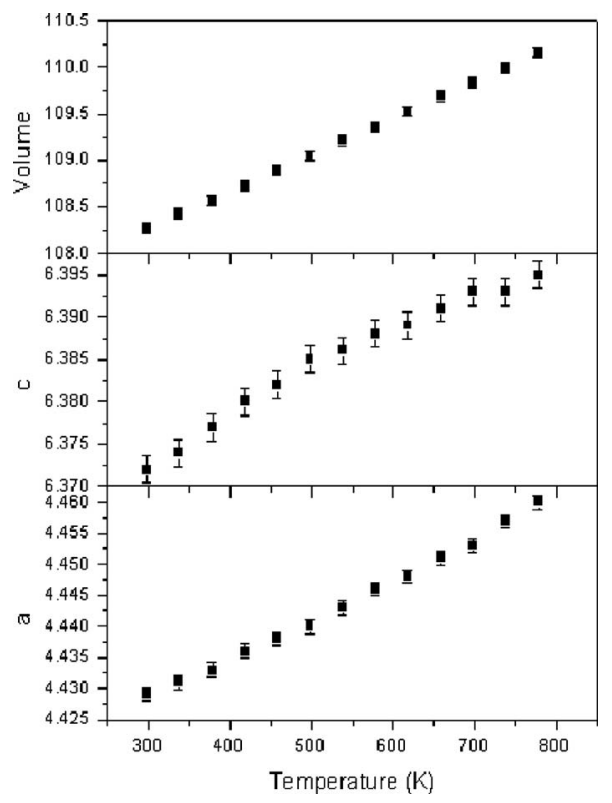

FIG. 2. Variation in $a$ and $c$ lattice parameters and cell volume from RT to $800 \mathrm{~K}$ for $\mathrm{CrSi}_{2}$. 


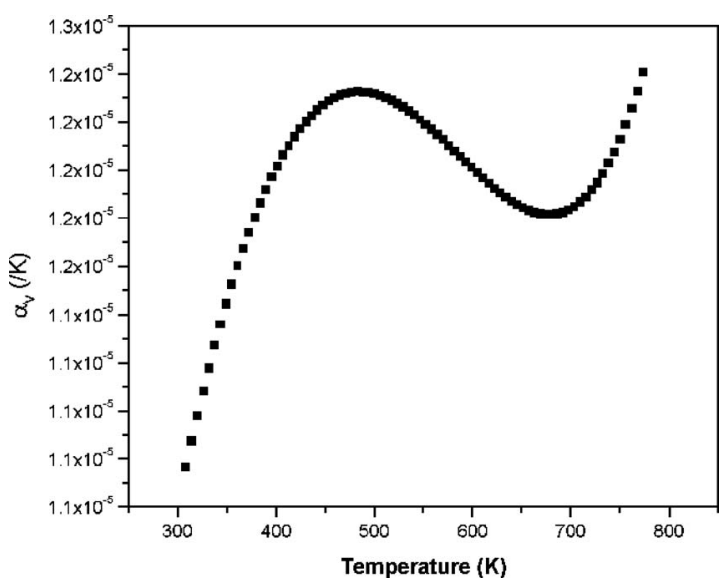

FIG. 3. The change in the volumetric $\alpha$ with temperature for $\mathrm{CrSi}_{2}$. An anomalous decrease in the $\alpha$ coefficient is observed in the temperature range of $400-650 \mathrm{~K}$.

the expansion values between the two crystallographic axes in this material. The average thermal expansion data (Fig. 3) also shows a decrease between 450 and $600 \mathrm{~K}$ (a polynomial fit was done to the volume versus temperature data and the average thermal expansion was then calculated from the derivative of polynomial curve). The $c / a$ ratio (Table II) shows a continuous decrease from 1.438 at room temperature to 1.433 at $800 \mathrm{~K}$.

\section{Thermal stability and microstructure}

The thermal stability of the synthesized powder in an ambient atmosphere is investigated by thermogravimetric analysis (TGA) as shown in Fig. 4. It is seen that the $\mathrm{CrSi}_{2}$ powder is thermally stable in the temperature range of $300-900 \mathrm{~K}$. The thermal stability of $\mathrm{CrSi}_{2}$ is attributed to the formation of a protective scale of chromium oxide in this temperature range, ${ }^{6,20}$ which prevents further oxidation of the material.

In Fig. 5 the SEM fractograph of the arc melted and hot pressed $\mathrm{CrSi}_{2}$ sample is shown. The average grain size is seen to be $>10 \mu \mathrm{m}$. The microstructure seems to be dense which was also confirmed from the density measurements of pressed pellets ( $>98 \%$ theoretical density). This is particu-

TABLE II. Refined lattice parameters and volume of $\mathrm{CrSi}_{2}$ as a function of temperature.

\begin{tabular}{ccccc}
\hline \hline Temperature $(\mathrm{K})$ & $a(\AA)$ & $c(\AA)$ & $c / a$ & Volume $\left(\AA^{3}\right)$ \\
\hline 298 & $4.429(1)$ & $6.372(1)$ & 1.4387 & $108.28(1)$ \\
338 & $4.431(1)$ & $6.374(1)$ & 1.4385 & $108.42(1)$ \\
378 & $4.433(1)$ & $6.377(1)$ & 1.4385 & $108.57(1)$ \\
418 & $4.436(1)$ & $6.380(1)$ & 1.4382 & $108.73(2)$ \\
458 & $4.438(1)$ & $6.382(1)$ & 1.4380 & $108.89(2)$ \\
498 & $4.440(1)$ & $6.385(1)$ & 1.4380 & $109.05(2)$ \\
538 & $4.443(1)$ & $6.386(1)$ & 1.4373 & $109.21(3)$ \\
578 & $4.446(1)$ & $6.388(1)$ & 1.4368 & $109.37(3)$ \\
618 & $4.448(1)$ & $6.389(2)$ & 1.4363 & $109.53(4)$ \\
658 & $4.451(1)$ & $6.391(1)$ & 1.4358 & $109.69(4)$ \\
698 & $4.453(1)$ & $6.393(2)$ & 1.4356 & $109.84(5)$ \\
738 & $4.457(1)$ & $6.393(2)$ & 1.4343 & $109.99(5)$ \\
778 & $4.460(1)$ & $6.395(3)$ & 1.4338 & $110.16(6)$ \\
\hline \hline
\end{tabular}

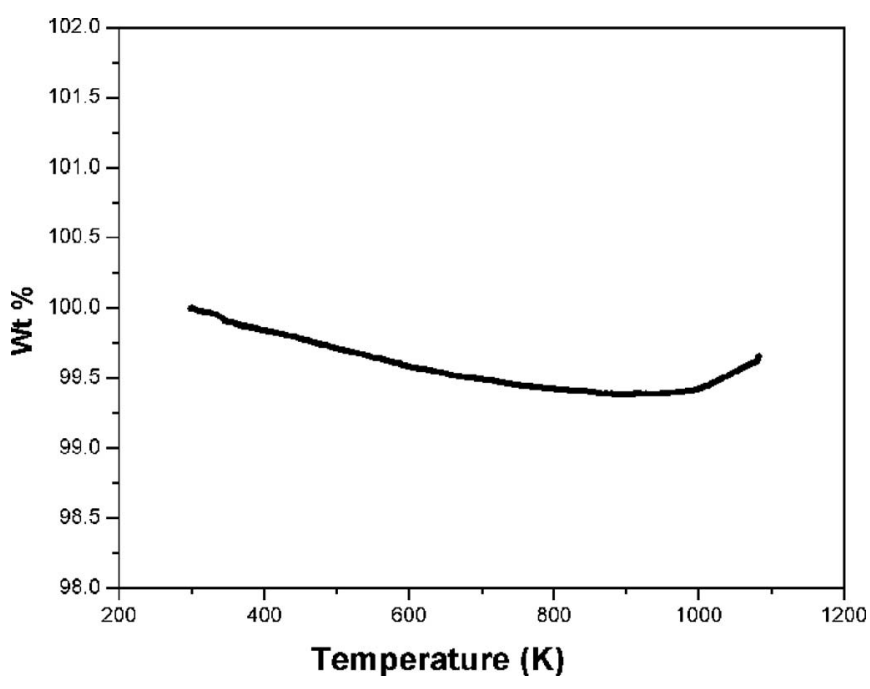

FIG. 4. TGA plot of $\mathrm{CrSi}_{2}$ as a function of temperature.

larly important in the determination of the $K$ value. A lower density can lead to a reduction of the $K$ value and hence an error in the calculation of ZT.

\section{Thermoelectric property measurement}

The $S, \rho$, and $K$ values for the hot pressed pellets were measured as a function of temperature. The plots of $\rho$ and $S$ are shown in Fig. 6. The electrical resistivity initially increases up to $550 \mathrm{~K}$ above which it decreases with increasing temperature. This behavior has been explained in the literature to be due to the degenerate semiconducting nature of $\mathrm{CrSi}_{2}{ }^{5}$ The initial region, where $\rho$ increases with temperature, is the mobility driven exhaustion range. Above $600 \mathrm{~K}$ the material enters its intrinsic regime and $\rho$ starts to decrease with increasing temperature. A similar trend is observed in the $S$ value with a maximum of $155 \mu \mathrm{V} / \mathrm{K}$ around $600 \mathrm{~K}$.

The thermal conductivity data which were measured by the laser flash technique are shown in Fig. 7. The specific heat data show a steady increase with temperature while the

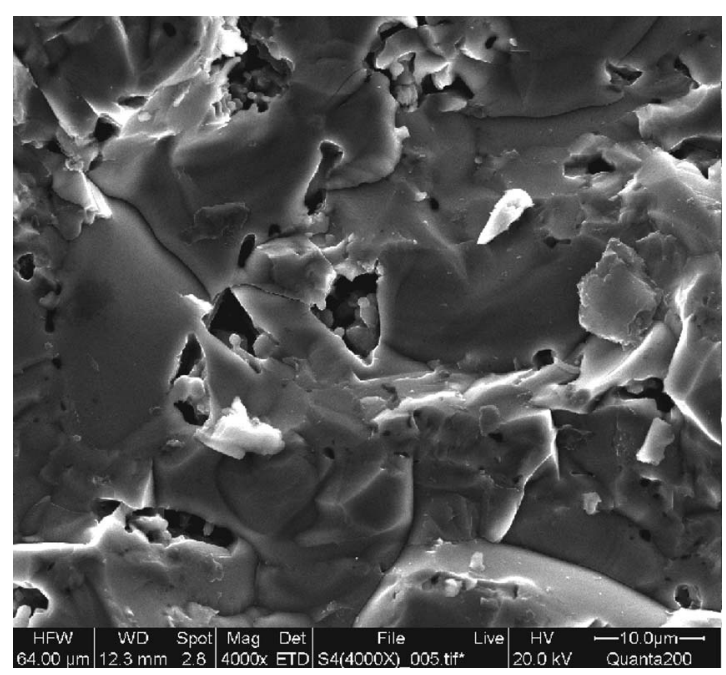

FIG. 5. The SEM taken on a fractured surface showing the dense microstructure. 
(a)
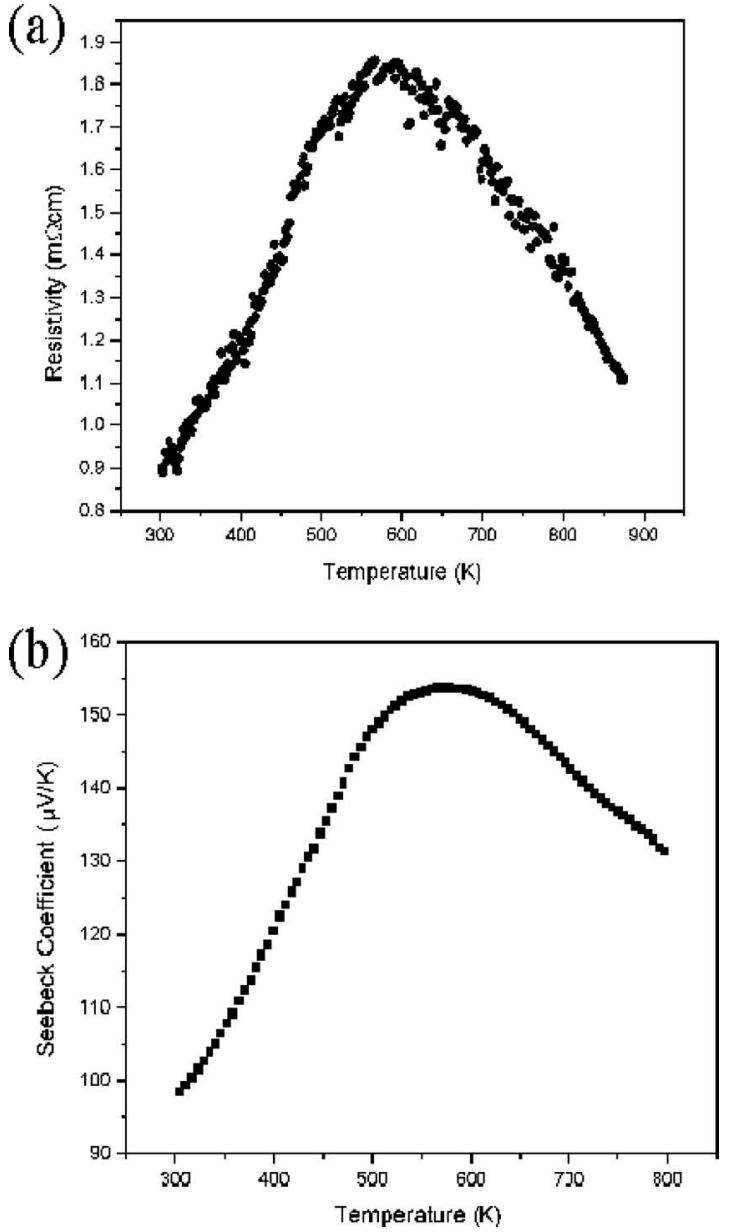

FIG. 6. (a) Electrical resistivity as a function of temperature. (b) Seebeck coefficient as a function of temperature.

diffusivity value has an opposite trend. The $K$ value [Fig. $7(\mathrm{c})]$ decreases with increasing temperature with the values ranging from 10 to $6 \mathrm{~W} / \mathrm{mK}$. This behavior is typical of any material at intermediate temperatures. ${ }^{21}$ The dimensionless figure of merit (ZT), plotted using Eq. (1), is shown in Fig. 8. The ZT value is seen to increase with temperature with a maximum of around 0.18 . Though this value is not very high, it is comparable to other thermoelectric silicides, i.e., $\beta$ - $\mathrm{FeSi}_{2}$ and $\mathrm{MnSi}_{1.75}$ in this temperature range. ${ }^{22,23}$

\section{COMPUTATIONS AND RESULTS}

Besides the early work of Mattheiss, ${ }^{12}$ recent theoretical investigations ${ }^{24,25}$ have focused on the structure stability of $\mathrm{CrSi}_{2}$. However, in view of the accurate determination of the crystal cell constants and position parameters of $\mathrm{Si}$ from x-ray refinement, it seemed relevant to further discuss the electronic band structure of this material. Furthermore, we provide an original description of the chemical bonding properties.

\section{A. Calculation methods}

Self-consistent calculations were carried out in the framework of the well established density functional theory. ${ }^{26}$ They are $a b$ initio in so far as the only input parameters are the crystal lattice specifications from x-ray diffrac-
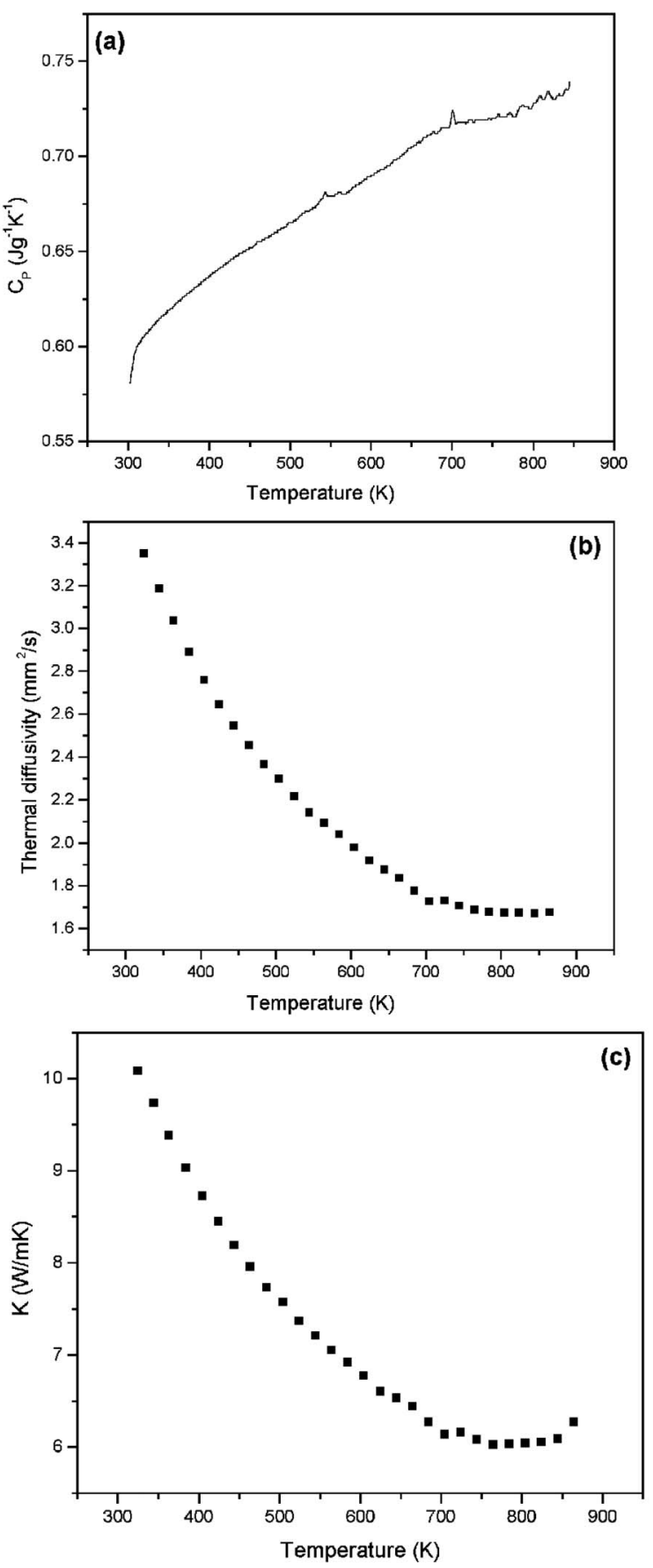

FIG. 7. (a) Specific heat data of $\mathrm{CrSi}_{2}$ as a function of temperature, (b) thermal diffusivity as a function of temperature from the laser flash technique, and (c) the calculated thermal conductivity $(K)$ as a function of temperature for pure $\mathrm{CrSi}_{2}$.

tion (XRD). We have mainly used the all electrons augmented spherical wave (ASW) method. ${ }^{27}$ The exchangecorrelation effects were accounted for within the generalized gradient approximation (GGA) using the parametrization of Perdew et ll $^{28}$ In the ASW method, the wave function is expanded in atom-centered augmented spherical waves, which are spherical Hankel functions and numerical solutions of Schrödinger's equation, respectively, outside and inside the so-called augmentation spheres. The Brillouin zone integration was carried out by increasing the $k$-mesh up to high precision sampling so that the energy and charge differ- 


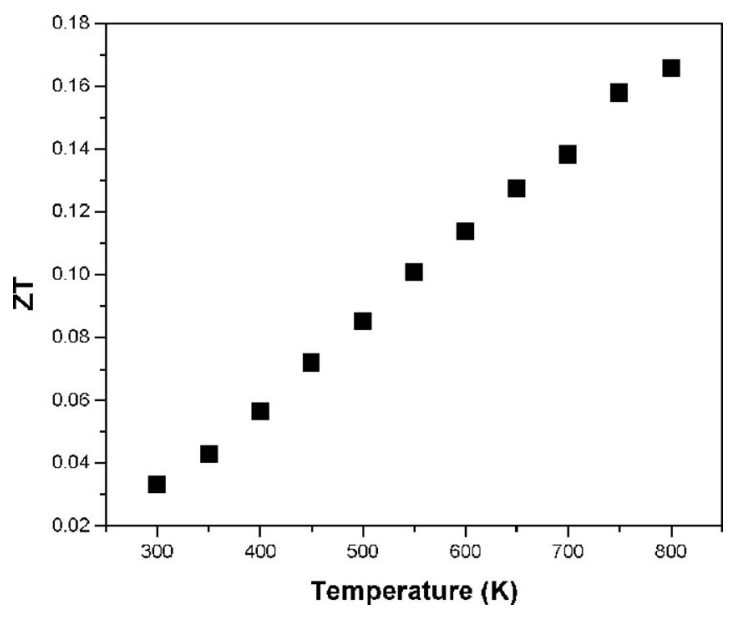

FIG. 8. The dimensionless figure of merit (ZT) of $\mathrm{CrSi}_{2}$ as a function of temperature.

ences between successive iterations converged below $\Delta E$ $=10^{-8}$ Ry $(1$ Ry and $13.6 \mathrm{eV})$ and $\Delta Q=10^{-8}$.

To extract more information about the nature of the interactions between the atomic constituents from electronic structure calculations, the crystal orbital overlap population ${ }^{29}$ (COOP) or the crystal orbital Hamiltonian population ${ }^{30}$ (COHP) may be employed. Both approaches provide a qualitative description of the bonding, nonbonding, and antibonding interactions between two atoms. A slight refinement of the COHP was recently proposed in the form of the "energy of covalent bond" (ECOV), which combines COHP and $\mathrm{COOP}$ to calculate quantities independent of the choice of the zero of potential. ${ }^{31}$ Both COOP and ECOV give similar general trends, but COOP, when defined within plane-wave basis sets, exaggerates the magnitude of antibonding states. In the present work, the ECOV was used for the chemical bonding analysis. In the plots, negative, positive, and zero unitless ECOV magnitudes are relevant to bonding, antibonding, and nonbonding interactions, respectively.

\section{B. Calculation results and discussion}

From the calculations carried out with high precision sampling in $k$ points of the Brillouin zone (BZ), we obtain the site projected density of states DOS (PDOS), the electronic band structure along the major directions of the hexagonal BZ, and the chemical bonding. Figure 9(a) shows the PDOS accounting for site multiplicity within the $\mathrm{CrSi}_{2}(Z$ $=3$ ) lattice. Along $x$ axis, the energy is with respect to the top of the valence band $\left(E_{V}\right)$, not the Fermi level, since the system can be identified as a semiconductor from the small energy separation between the valence band and the conduction band $(\mathrm{CB})$. The valence band (VB) is dominated at the lower energy part from -15 to $-1 \mathrm{eV}$ by $\mathrm{Si} s, p$ states which resemble the large dispersion of the DOS. The $\mathrm{Cr}$ and Si PDOS in this energy range have a similar skyline so that one expects a quantum mixing between $\mathrm{Si} s, p$ and the itinerant part of the $\mathrm{Cr}$ valence states. This is illustrated below by the chemical bonding ECOV plots. A relevant feature is the presence of large intensity Cr d PDOS on both sides of the top of the VB and lower part of the CB. These states, which mix
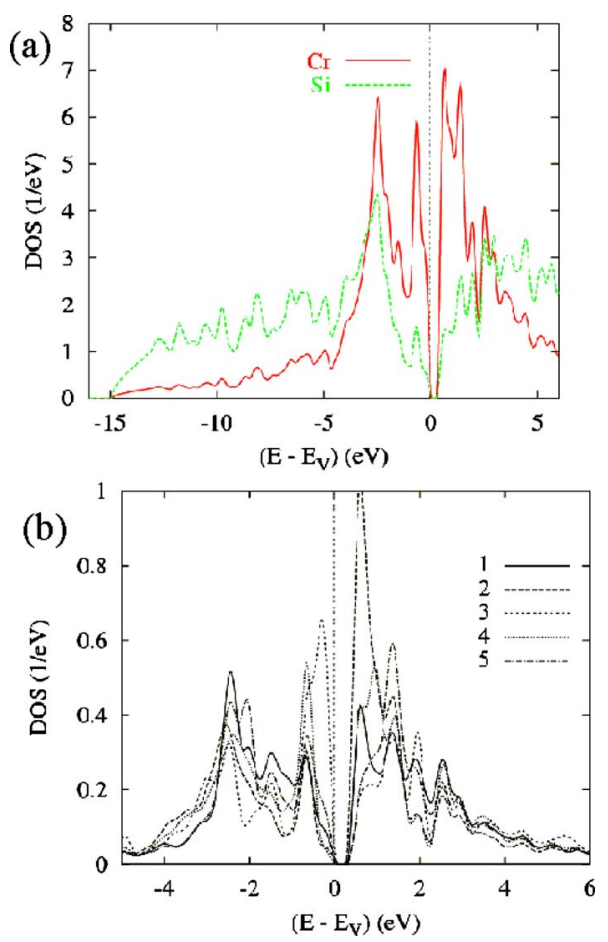

FIG. 9. (Color online) (a) Site projected density of states (PDOS) of $\mathrm{CrSi}_{2}$ $(Z=3)$, (b) crystal field analysis of the Cr PDOS; the $5 d$ orbitals labels are as follows: (1) $d_{x y}$, (2) $d_{y z}$, (3) $d_{z 2}$, (4) $d_{x z}$, and (5) $d_{x 2-y 2}$.

little with Si states in the close neighborhood of $E_{V}$, should have a nonbonding behavior. In order to clearly identify their nature, a projection of the $\mathrm{Cr} 5 d$ orbitals is shown in Fig. 9(b). From this analysis, it appears that the major contributions to the Cr PDOS at the VB top and CB bottom arise from the $d_{z 2}$ and $d_{y z}$ manifolds, i.e., those orbitals involved with the $k_{z}$ direction of the BZ. This is further illustrated by the plot of the band structure of $\mathrm{CrSi}_{2}$ at Figs. 10(a)-10(c) in a narrow energy window around $E_{V}$. The gap is seen to be indirect: $L_{V}-M_{C}$ with a $\sim 0.35 \mathrm{eV}$ magnitude, in agreement with former computations. ${ }^{12}$ The fat bands in Figs. 10(b) and 10(c) are meant to stress the role played by $\mathrm{Cr} d$ orbitals in the above analysis on both sides of the gap in Fig. 9(b). Despite the covalent nature of the quantum mixing between $\mathrm{Cr}$ and $\mathrm{Si}$ valence states, it can be assumed that the major bands at the top of VB are $\mathrm{Cr} d_{z 2}$ [Fig. 10(b)] and $\mathrm{Cr} d_{y z}$ at the bottom of the CB.

In Figure 11, an analysis of the chemical bonding is provided using the ECOV criterion described above. Along the $y$ axis negative, positive, and zero magnitudes point to bonding, antibonding, and nonbonding interactions, respectively; the interactions are atom-to-atom, involving one species of each kind. Within the VB the major contribution is found to be due to the bonding $\mathrm{Cr}-\mathrm{Si}$ ECOV followed by a smeared $\mathrm{Si}-\mathrm{Si}$ bonding and a small $\mathrm{Cr}-\mathrm{Cr}$ metallic bond. The $\mathrm{Cr}-\mathrm{Cr}$ ECOV becomes antibonding on both sides of the top of the valence band indicating no quantum mixing of the $d_{z}^{2}$ and $d_{y Z}$ of $\mathrm{Cr}$ [see Fig. 9(b)] with that of Si. The total ECOV (shown in inset of Fig. 11) becomes antibonding in the range of $-1 \mathrm{eV}$ to the $\mathrm{VB}$ edge. Also, there is a small bonding region above the VB. This feature may be responsible for the decrease in $\alpha_{v}$ observed in Fig. 3, i.e., on increasing tempera- 

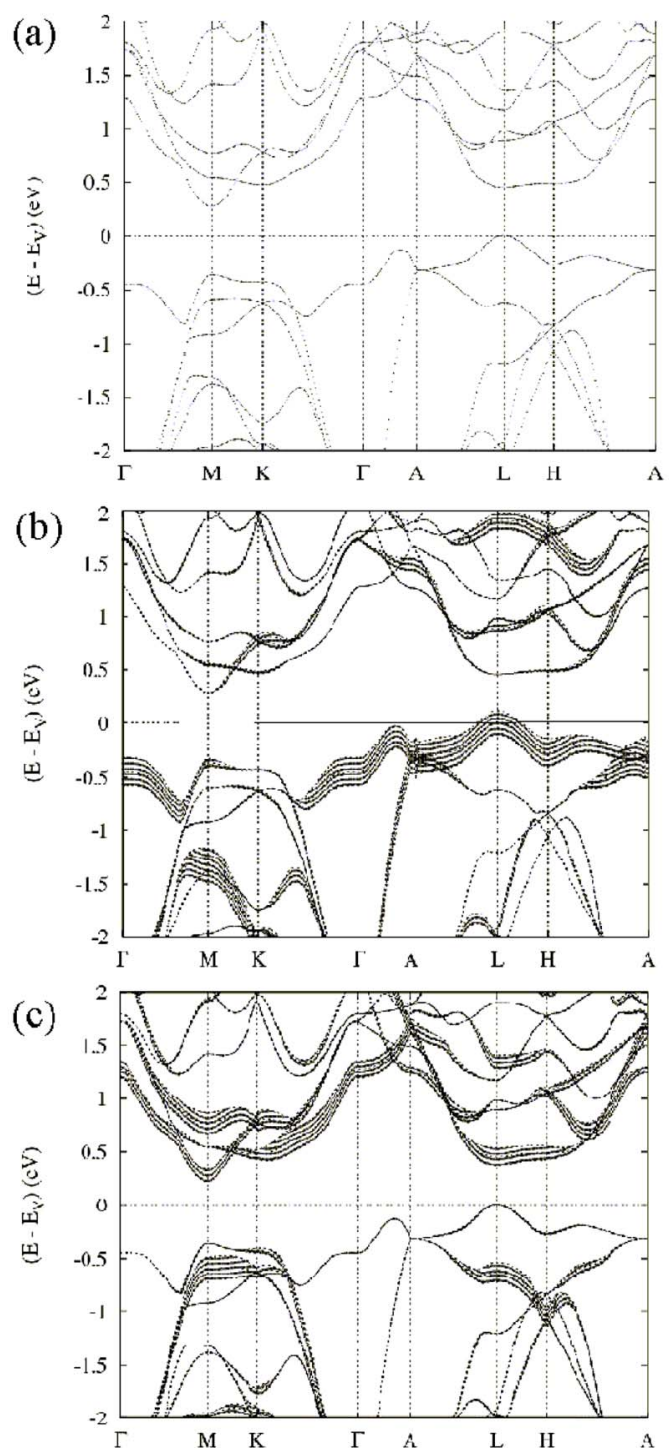

FIG. 10. (Color online) (a) The band structure of $\mathrm{CrSi}_{2}(Z=3)$ in a narrow window around the top of the valence band $E_{V}$. The indirect band gap $\left(L_{V}-M_{C}\right)$ can be observed. (b) Fat band representation of $\mathrm{Cr} 3 d_{z 2}$ orbital. (c) Fat band representation of $\mathrm{Cr} 3 d_{y z}$ orbital.

ture; electrons from the antibonding states just below the top of $E_{V}$ go to the bonding states above. This causes an increase in the bond strength and thereby reduces the increase in $\alpha_{V}$. The $\mathrm{CB}$ is dominated by antibonding $\mathrm{Cr}-\mathrm{Si}$ and bonding $\mathrm{Cr}-\mathrm{Cr}$ interactions. Thus chemical bonding within the chromium silicide system is a delicate interplay between bonding and antibonding behaviors, which are very likely to change when lattice expansions are provoked.

\section{CONCLUSION}

The structure refinement of $\mathrm{CrSi}_{2}$ was carried out by the Rietveld method considering $\mathrm{Pb}_{2} 22$ as the space group. The refined lattice parameters are $a=4.42758$ (7) and $c$ $=6.36805$ (11). The $x$ positional parameter of $\mathrm{Si}$ is shifted from its ideal position to $0.165591(1)$. The calculation of lattice parameters as a function of temperature indicates a nonlinear increase in the $c$ lattice value. This is also reflected in the average thermal expansion value which shows a dip in

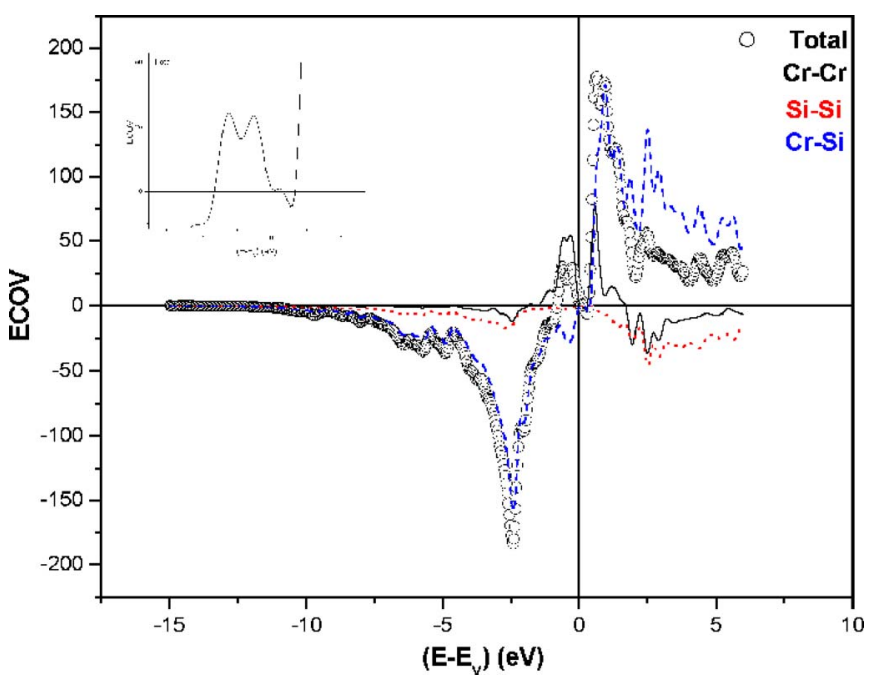

FIG. 11. (Color online) The chemical bonding from ECOV criterion for two-atom interactions within $\mathrm{CrSi}_{2}$. The total ECOV near the band gap is shown in inset.

the temperature range of 450-600 K. The temperature range also corresponds to the inversion in the electrical resistivity and Seebeck values. Thermal conductivity decreases with increasing temperature from 10 to $6 \mathrm{~W} / \mathrm{mK}$ to. ZT increases with temperature with a maximum value of 0.18 . The band structure analyes within the density functional theory (DFT) from the plots of the DOS, band structure, and chemical bonding allow us to identify the role of the chemical constituents within the VB and around the gap.

${ }^{1}$ A. K. Vasudevan and J. J. Petrovic, Mater. Sci. Eng., A 155, 1 (1992).

${ }^{2}$ C. B. Vining, CRC Handbook of Thermoelectrics (CRC, New York, 1995), Chap. 23.

${ }^{3}$ T. Kajikawa, K. Shiraishi, T. Sugiyama, M. Omori, and T. Hirai, "Thermoelectric properties of high temperature transition metal silicides prepared by spark plasma sintering method," 18th International Conference on Thermoelectrics, pp. 601 (1999).

${ }^{4}$ T. Kajikawa, T. Sugiyama, M. Serizawa, K. Kamio, M. Koike, T. Ohta, and M. Omori, "Thermoelectric properties and electrode bonding performance for metal silicides," 20th International Conference on Thermoelectrics, pp. 236 (2001).

${ }^{5}$ I. Nishida and T. Sakata, J. Phys. Chem. Solids 39, 499 (1978).

${ }^{6}$ J. Ma, Y. Gu, L. Shi, L. Chen, Z. Yang, and Y. Oian, J. Alloys Compd. 376, 176 (2004).

${ }^{7}$ P. Villars and L. D. Calvert, Pearson's handbook of Crystallographic Data for Intermetallic Phases (American Society for Metals, Metals Park, 1985).

${ }^{8}$ L. F. Mattheiss, Phys. Rev. B 45, 3252 (1992).

${ }^{9}$ Y. Harada, M. Morinaga, D. Saso, T. Takata, and M. Sakata, Intermetallics 6, 523 (1998).

${ }^{10}$ M. C. Bost and J. E. Mahan, J. Appl. Phys. 83, 839 (1988).

${ }^{11}$ A. V. Krivosheeva, V. L. Shaposhnikov, and V. E. Borisenko, Mater. Sci. Eng., B 101, 309 (1991).

${ }^{12}$ L. F. Mattheiss, Phys. Rev. B 43, 12549 (1991).

${ }^{13}$ H. Hohl, A. P. Ramirez, T. T. M. Palstra, and E. Bucher, J. Alloys Compd. 248, 70 (1997).

${ }^{14}$ T. Dasgupta and A. M. Umarji, "Role of milling parameters and impurity on the thermoelectric properties of mechanically alloyed chromium silicide," J. Alloys Compd. (in press).

${ }^{15}$ Z. J. Pan, L. T. Zhang, and J. S. Wu, Scr. Mater. 56, 245 (2007).

${ }^{16}$ Z. J. Pan, L. T. Zhang, and J. S. Wu, Scr. Mater. 56, 257 (2007).

${ }^{17}$ A. C. Larson and R. B. Von Dreele, Los Alamos National Laboratory Report No. LAUR 86-748 (Los Alamos National Laboratory, Los Alamos, NM, 2000).

${ }^{18}$ W. Lasocha and K. Lewinski, J. Appl. Crystallogr. 27, 437 (1994).

${ }^{19}$ P. Dordor, E. Marquestaut, and G. Villeneuve, Rev. Phys. Appl. 15, 1607 
(1980).

${ }^{20}$ A. Tomasi, R. Ceccato, M. Nazmy, and S. Gialanella, Mater. Sci. Eng., A 239-240, 877 (1997).

${ }^{21}$ C. Kittel, Introduction to Solid State Physics (Wiley, New York, 1995).

${ }^{22}$ U. Birkholz, E. Grop, and U. Stohrer, CRC Handbook of Thermoelectrics (CRC, New York, 1995), Chap. 24.

${ }^{23} \mathrm{~K}$. Zaitsev CRC Handbook of Thermoelectrics (CRC, New York, 1995), Chap. 25.

${ }^{24}$ G. A. Ackland, Phys. Rev. B 71, 012106 (2005).

${ }^{25}$ D. A. Pankhurst, D. Nguyen-Manh, and D. G. Pettifor, Phys. Rev. B 69, 075113 (2004)
${ }^{26}$ P. Honenberg and W. Kohn, Phys. Rev. 136, B864 (1964); W. Kohn and L. J. Sham, Phys. Rev. 140, A1133 (1965).

${ }^{27}$ A. R. Williams, J. Kübler, and C. D. Gelatt, Phys. Rev. B 19, 6094 (1979); V. Eyert, The Augmented Spherical Wave Method: A Comprehensive Treatment (Springer, Heidelberg, 2007).

${ }^{28}$ J. P. Perdew, K. Burke, and M. Ernzerhof, Phys. Rev. Lett. 77, 3865 (1996).

${ }^{29}$ R. Hoffmann, Angew. Chem., Int. Ed. Engl. 26, 846 (1987).

${ }^{30}$ R. Dronskowski and P. E. Blöchl, J. Phys. Chem. 97, 8617 (1993).

${ }^{31}$ N. Börnsen, B. Meyer, O. Grotheer, and M. Fähnle, J. Phys.: Condens. Matter 11, L287 (1999). 